\title{
510(k) Summary
}

This summary of $510(k)$ safety and effectiveness information is being submitted in accordance with the requirement of SMDA and 21 CFR 807.92.

\section{0 submitter's information}

Name:

Address:

Phone number:

Fax number:

Contact:

Date of Application:
Andon Health Co., Ltd.

No 3, Jinping Street Ya An Road, Nankai District, Tianjin, P.R. China

\subsection{Device information}

Trade name:

Device name:

Classification name: Noninvasive blood pressure measurement system
iHealth BP7-Wireless Blood Pressure Wrist Monitor KD-972 Fully Automatic Wireless Blood Pressure Wrist Monitor

\section{Liu Yi}

05/08/2012

\subsection{Classification}

Production code: DXN- Noninvasive blood pressure measurement system.

Regulation number: 870.1130

Classification: ' II

Panel: $\quad$ Cardiovascular

\subsection{Predicate device information}

Manufacturer: Andon Health Co., Ltd.

Device: $\quad$ KD-7964 Fully Automatic Electronic Blood Pressure Monitor

510(k) number: K102906

\subsection{Device description}

KD-972 Wireless Blood Pressure Monitor is for use by medical professionals or at home and is a non-invasive blood pressure measurement system 
intended to measure the diastolic and systolic blood pressures and pulse rate of an adult individual by using a non-invasive technique in which an inflatable cuff is wrapped around the wrist. The cuff circumference is limited to $13.5 \mathrm{~cm}-22 \mathrm{~cm}$.

It is designed and manufactured according to IEC 80601-2-30- Particular requirements for the basic safety and essential performance of automated non-invasive sphygmomanometers.

The operational principle is based on oscillometric and silicon integrates pressure sensor technology. it can calculate the systolic and diastolic blood pressure, the measurement results can also be classified by the function of blood pressure classification indicator. If any irregular heartbeat is detected, it can be shown to the user. More over, it also obtains the function of averaging the measurement results.

KD-972 Fully Automatic Wireless Blood Pressure Wrist Monitor achieves its function by integrate the device with an iPhone, iPod touch or iPad. For it does not contain an LCD or other display components, so It's necessary for the new device to connect to an iPhone, iPod touch or iPad containing a support software to constitute a complete blood pressure measurement system. And the new device connect iPhone, iPod or iPad through bluetooth.

\subsection{Intended use}

KD-972 Fully Automatic Wireless Blood Pressure Wrist Monitor is for use by medical professionals or at home and is a non-invasive blood pressure measurement system intended to measure the diastolic and systolic blood pressures and pulse rate of an adult individual by using a non-invasive technique in which an inflatable cuff is wrapped around the wrist. The cuff circumference is limited to $13.5 \mathrm{~cm}-22 \mathrm{~cm}$.

The intended use and the indication for use of KD-972, as described in the labeling are the same as its predicate device KD-7964.

\subsection{Summary comparing technological characteristics with predicate device}

\begin{tabular}{|l|l|}
\hline Technological Characteristics & Comparison result \\
\hline Design principle & Identical \\
\hline Appearance & Similar \\
\hline Patients contact Materials & Identical \\
\hline Performance & Similar \\
\hline
\end{tabular}




\begin{tabular}{|l|l|}
\hline Biocompatibility & Identical \\
\hline Mechanical safety & Identical \\
\hline Energy source & Identical \\
\hline Standards met & Identical \\
\hline Electrical safety & Identical \\
\hline EMC & Identical \\
\hline Function & Similar \\
\hline
\end{tabular}

\subsection{Performance summary}

KD-972 Fully Automatic Wireless Blood Pressure Wrist Monitor conforms to the following standards:

- IEC 60601-1, Medical Electrical Equipment - Part 1: General Requirements for Safety, 1988; Amendment 1, 1991-11, Amendment 2, 1995.

- EN 60601-1-2, Medical Electrical Equipment - Part 1-2: General Requirements for Safety - Collateral standard: Electromagnetic Compatibility - Requirements and Tests, 2007.

- IEC 80601-2-30, Medical electrical equipment-Part 2-30:Particular requirements for the basic safety and essential performance of automated non-invasive sphygmomanmeters, 2009 .

\subsection{Comparison to the predicate device and the conclusion}

Our device KD-972 Fully Automatic Wireless Blood Pressure Wrist Monitor is substantially equivalent to the Fully Automatic Electronic Blood Pressure Monitor KD-7964 whose $510(\mathrm{k})$ number is K102906.

KD-972 and KD-7964 are very similar in the intended use, the design principle, the performance and the applicable standards. Only their appearance, the memory time, the average function are different. The measure process is also changed, that is the new device will get the measurement results when the device is inflating, while KD -7964 gets the result during the deflating period. The data transfer method of the new device KD-972 is changed to Bluetooth and the data displayed on iPhone, while KD-7964 transfer the data to PC while receive available command.

However, the test in this submission provides demonstration that these small differences do not raise any new questions of safety and effectiveness 
Andon Health Co., Ltd.

c/o Ms. Liu Yi

President

No. 3 Jin Ping Street, Ya An Road, Nankai District

Tianjin

China 300190

\section{Re: K121470}

Trade/Device Name: Fully Automatic Wireless Blood Pressure Wrist Monitor, KD-972

Regulatory Number: 21 CFR 870.1130

Regulation Name: Non-invasive Blood Pressure Measurement System

Regulatory Class: II (two)

Product Code: DXN

Dated: May 14, 2012

Received: May 17, 2012

Dear Ms. Yi:

We have reviewed your Section $510(\mathrm{k})$ premarket notification of intent to market the device referenced above and have determined the device is substantially equivalent (for the indications for use stated in the enclosure) to legally marketed predicate devices marketed in interstate commerce prior to May 28, 1976, the enactment date of the Medicál Device Amendments, or to devices that have been reclassified in accordance with the provisions of the Federal Food, Drug, and Cosmetic Act (Act) that do not require approval of a premarket approval application (PMA). You may, therefore, market the device, subject to the general controls provisions of the Act. The general controls provisions of the Act include requirements for annual registration, listing of devices, good manufacturing practice, labeling, and prohibitions against misbranding and adulteration. Please note: CDRH does not evaluate information. related to contract liability warranties. We remind you, however, that device labeling must be truthful and not misleading.

If your device is classified (see above) into either class II (Special Controls) or class III (PMA), it may be subject to additional controls. Existing major regulations affecting your device can be 
Page 2 - Ms. Liu Yi

found in the Code of Federal Regulations, Title 21, Parts 800 to 898. In addition, FDA may publish further announcements concerning your device in the Federal Register.

Please be advised that FDA's issuance of a substantial equivalence determination does not mean that FDA has made a determination that your device complies with other requirements of the Act or any Federal statutes and regulations administered by other Federal agencies. You must comply with all the Act's requirements, including, but not limited to: registration and listing (21 CFR Part 807); labeling (21 CFR Part 801); medical device reporting (reporting of medical device-related adverse events) (21 CFR 803); good manufacturing practice requirements as set forth in the quality systems (QS) regulation (21 CFR Part 820); and if applicable, the electronic product radiation control provisions (Sections 531-542 of the Act); 21 CFR 1000-1050.

If you desire specific advice for your device on our labeling regulation (21 CFR Part 801), please go to http://www.fda.gov/AboutFDA/CentersOffices/CDRH/CDRHOffices/ucm 1 15809.htm for the Center for Devices and Radiological Health's (CDRH's) Office of Compliance. Also, please note the regulation entitled, "Misbranding by reference to premarket notification". (21CFR Part 807.97). For questions regarding the reporting of adverse events under the MDR regulation (21 CFR Part 803), please go to

http://www.fda.gov/MedicalDevices/Safety/ReportaProblem/default.htm for the CDRH's. Office of Surveillance and Biometrics/Division of Postmarket Surveillance.

You may obtain other general information on your responsibilities under the Act from the Division of Small Manufacturers, International and Consumer Assistance at its toll-free number (800) 638-2041 or (301) 796-7100 or at its Internet address http://www.fda.gov/MedicalDevices/ResourcesforYou/Industry/default.htm.

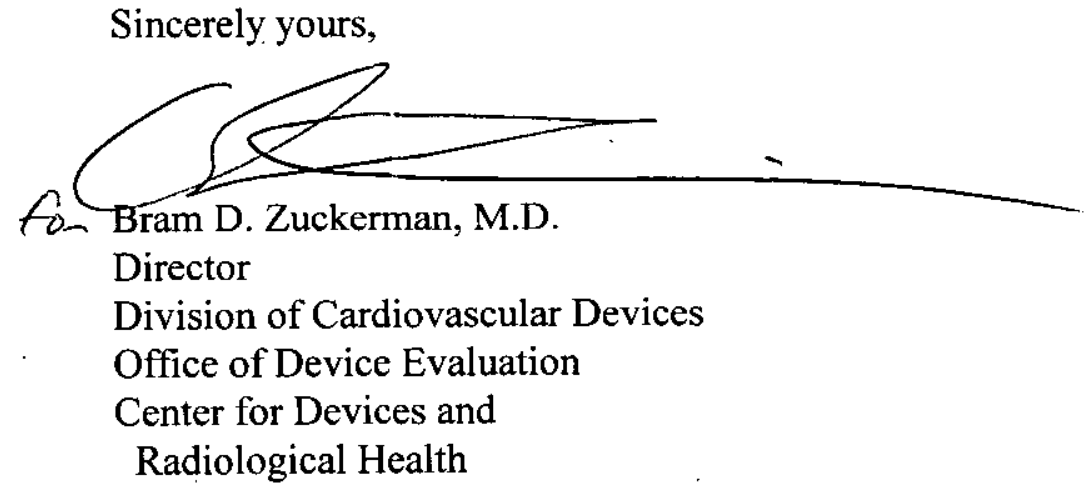

Enclosure 


\section{Statement of Indications for Use}

\section{0(k) Number :}

Device name:

KD-972 Fully Automatic Wireless Blood Pressure Wrist Monitor

\section{Indications for use:}

KD-972 Fully Automatic Wireless Blood Pressure Wrist Monitor is for use by medical professionals or at home and is a non-invasive blood pressure measurement system intended to measure the diastolic and systolic blood pressures and pulse rate of an adult individual by using a non-invasive technique in which an inflatable cuff is wrapped around the wrist. The cuff circumference is limited to $13.5 \mathrm{~cm}-22 \mathrm{~cm}$.

Prescription use

Part 21 CFR 801 Subpart D)
AND/OR Over-The-Counter Use YES

(21 CFR 807 Subpart C)

(PLEASE DO NOT WRITE BELOW THIS LINE-COUNTINUE ON ANOTHER PAGE IF NEEDED)

Concurrence of $\mathrm{CDRH}$, Office of Device Evaluation (ODE)

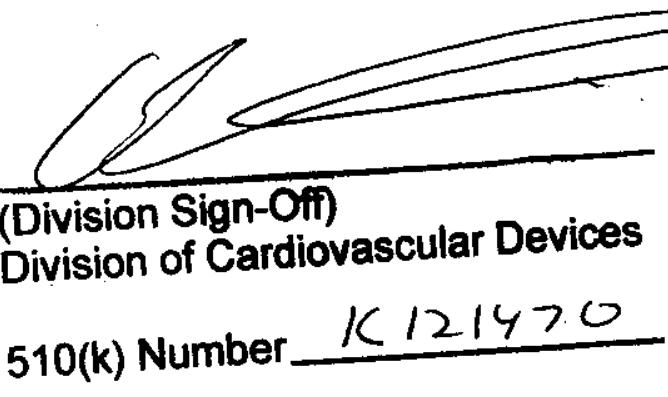

